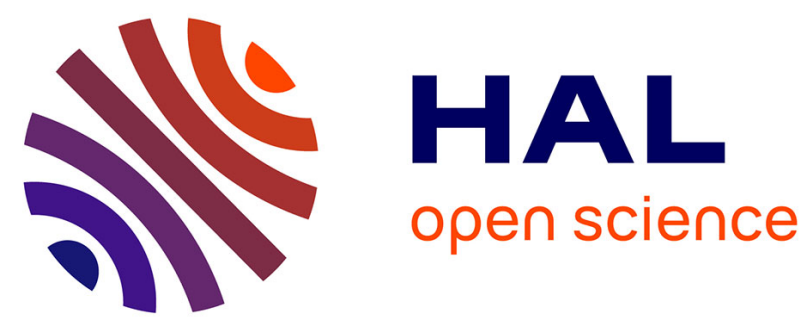

\title{
DC building management system with IEEE 802.3bt standard
}

Karem Hafsi, Denis Genon-Catalot, Jean-Marc Thiriet, Olivier Lefevre

\section{To cite this version:}

Karem Hafsi, Denis Genon-Catalot, Jean-Marc Thiriet, Olivier Lefevre. DC building management system with IEEE 802.3bt standard. HSPR 2021 IEEE International Conference on High Performance Switching and Routing (HSPR), Jun 2021, Paris, France. pp.1-8, 10.1109/HPSR52026.2021.9481806 . hal-03429857

\section{HAL Id: hal-03429857 https://hal.science/hal-03429857}

Submitted on 15 Nov 2021

HAL is a multi-disciplinary open access archive for the deposit and dissemination of scientific research documents, whether they are published or not. The documents may come from teaching and research institutions in France or abroad, or from public or private research centers.
L'archive ouverte pluridisciplinaire HAL, est destinée au dépôt et à la diffusion de documents scientifiques de niveau recherche, publiés ou non, émanant des établissements d'enseignement et de recherche français ou étrangers, des laboratoires publics ou privés. 


\title{
DC building management system with IEEE 802.3bt standard
}

\author{
Karem Hafsi \\ LCIS Laboratory \\ Univ. Grenoble Alpes \\ Valence, France
}

\author{
Denis Genon-Catalot \\ LCIS Laboratory \\ Univ. Grenoble Alpes \\ Valence, France
}

\author{
Jean-Marc Thiriet \\ GIPSA-lab Laboratory \\ Univ. Grenoble Alpes \\ Grenoble, France
}

Karem.Hafsi@ 1cis.grenoble-inp.fr denis.genon-catalot@ lcis.grenoble-inp.fr jean-marc.thiriet@univ-grenoble-alpes.fr

\author{
Olivier Lefevre \\ AI Departement \\ Ubiant \\ Lyon, France \\ olivier.lefevre@ubiant.com
}

\begin{abstract}
We present the results for a new building supply and communication architecture based on Power Over Ethernet (PoE) with the IEEE 802.3bt standard. Driven by technological advances like photovoltaic production, LED lighting terminals, computer terminals, tablets, screens and the advancement of power electronics that allow more and more conversions with very high efficiency, direct current in buildings might be the right solution to reduce energy consumption and go towards positive energy buildings. With the new IEEE 802.3bt PoE standard which allows $99 \mathrm{~W}$ and with the increasing demand for new services in smart buildings and the emerging of IoT devices, we propose a communication and supply architecture completely based on the IP protocol. This paper presents feedback of the first deployment of a smart building totally powered by $\mathrm{PoE}$ devices, we analyse the power supply architecture and the application communication protocol that we have used which is MQTT (Message Queuing Telemetry Transport).
\end{abstract}

Keywords-Smart Building, DC Bus architecture, PoE, IEEE 802.3bt, MQTT, TLS, BMS.

\section{INTRODUCTION}

Global climate change is one of the biggest issues we are facing nowadays. With the need of industrial sustainability, this issue has become more and more challenging, the growth of $\mathrm{CO} 2$ emission especially in the electrical production sector is one of the main factors of the global climate change; the growth had reached 13603 MT in the world by 2017 as shown in Fig 1. The global

978-1-6654-4005-9/21/\$31.00 (C) 2021 IEEE residential sector consumed more than 516595 ktoe in 2018 , or nearly $26 \%$ of the final electricity consumption as shown in Figure 2. Meanwhile, we may notice that the modern buildings have reached a certain connectivity level and have changed into industrial control systems by embedding IP-based technologies throughout buildings. This is used to connect sensors, controllers and supervisory software, which allows to better manage the energy consumption. The IoT field has well-progressed this last decade and at a time when wireless dominates, Ethernet may seem like an old technology, but with the new IEEE 802.3bt standard which allows $99 \mathrm{~W}$ of power to be transmitted over Ethernet cables we think that we should take into account this evolution and better exploit it in the building ecosystem. In order to achieve this, this project proposes a new DC nano-grid architecture for tertiary, commercial or office buildings, with a centralised management system based on a BMS (Building Management System) server and smart PoE switches to distribute the power supply and to associate in a most efficient way intermittent renewable sources of energy.

In this paper, we present in the first part our architecture based on the PoE IEEE 802.3bt standards, we explain our first deployment of a smart building which employs only one DC voltage $48 \mathrm{~V}$ that supply our smart PoE switches. We present also a new vision for managing the power supply of the installed devices in a way that reduces the global energy consumption. in the 
second part we present the communication architecture, the IoT application protocol MQTT (Message Queuing Telemetry Transport) and some tests and results about its performances.

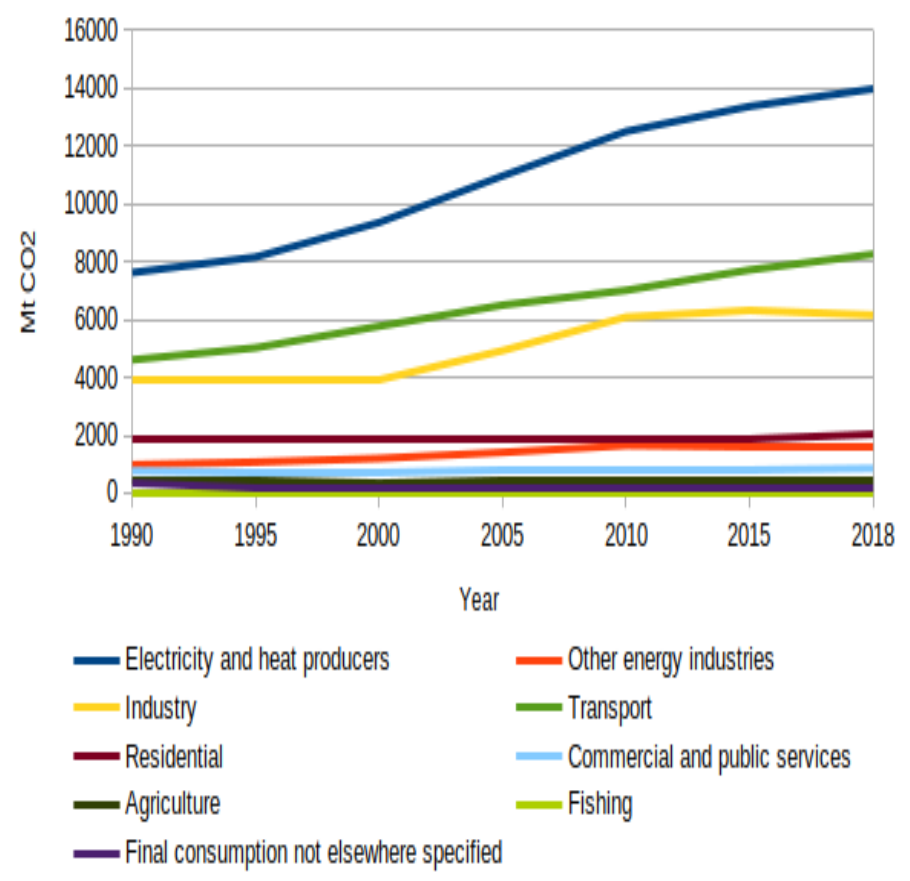

Fig. 1: CO2 emissions by sector, World 1990-2018[1]

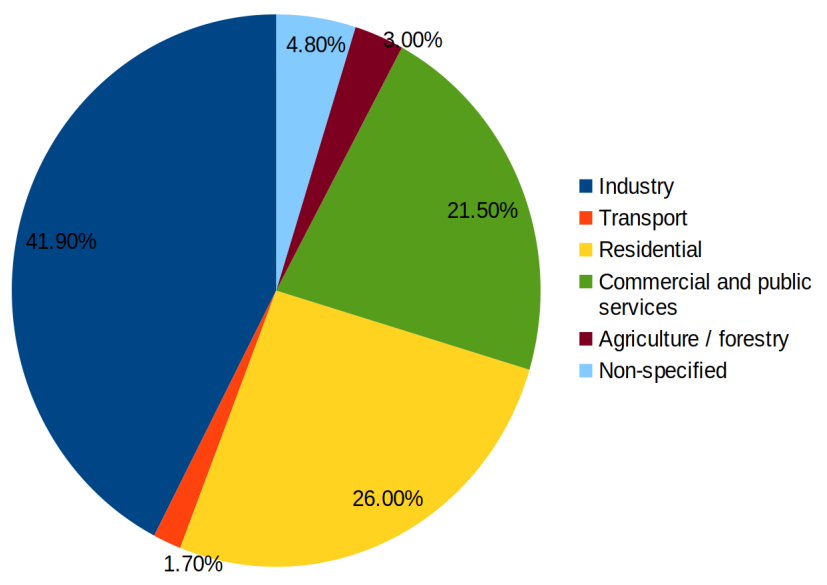

Fig. 2: Electricity final consumption by sector, 2018 [2]

\section{New Power SUPPly SyStem For SMART NANO-GRIDS}

\section{A. Introduction}

Many studies have been done on nano-grid architectures, most of those studies concern $\mathrm{AC}$ bus. In this section, we present a new power supply architecture based on AC bus and 48 VDC. The originality of our architecture relies on IEEE 802.3bt PoE standard which is used on a large scale to supply all the lighting, sensing and HVAC (Heating, Ventilation and Air-Conditioning) systems in the building. In the next subsections, we present the PoE technology within our architecture, the benefits, the disadvantages and some perspectives for this technology.

\section{B. PoE and the IEEE 802.3bt}

PoE is a technology that lets network cables carry electrical power over an existing data connection with a single CATx Ethernet cable.

PoE technology relies on the IEEE 802.3af, 802.3at and 802.3bt standards [3], which are set by the Institute of Electrical and Electronics Engineers (IEEE). PoE-capable devices can be Power Sourcing Equipment (PSE), Powered Device (PD), or sometimes both. The device that transmits power is a PSE, while the device that is powered is a PD. As the PoE technology has developed, the amount of power that can be sent over an Ethernet cable has increased. IEEE-compliant PoE switches and injectors can deliver from 15 watts to 99 watts of power per port. Table I. presents the various standards of the PoE: PoE technology has many advantages, the

\begin{tabular}{|l|l|l|l|}
\hline PoE Standard & $\begin{array}{l}\text { PoE } \\
\text { Common } \\
\text { Name }\end{array}$ & $\begin{array}{l}\text { Power Out- } \\
\text { put }\end{array}$ & Year \\
\hline IEEE 802.3af & PoE & $15.40 \mathrm{~W}$ & 2003 \\
\hline IEEE 802.3at & PoE+ & $30 \mathrm{~W}$ & 2009 \\
\hline $\begin{array}{l}\text { IEEE 802.3bt } \\
\text { Type 3 4PPoE }\end{array}$ & $\begin{array}{l}\text { Ultra PoE, } \\
\text { UPoE }\end{array}$ & $60 \mathrm{~W}$ & 2018 \\
\hline $\begin{array}{l}\text { IEEE 802.3bt } \\
\text { Type 4 Ultra PoE, }\end{array}$ & \begin{tabular}{l} 
UPoE \\
\hline
\end{tabular} & $100 \mathrm{~W}$ & 2018 \\
\hline
\end{tabular}

TABLE I: Evolution of the PoE Standards

remote installation generally costs less than for an optical fiber and does not require a qualified electrician, saving building owners valuable time and labor costs, further, the Ethernet cable used in PoE is often already installed in many commercial and residential buildings. In addition to that, PoE uses relatively low voltages, so it is safe 
and there is a low risk of electrical hazard. Furthermore PoE technology assures reliability and scalability, due to the fact that PoE devices are in full compliance with the IEEE 802.3 standard that defines the standards for Ethernet networks.

\section{Architecture and energy routing}

As mentioned in the introduction of this section, our architecture combine the use of AC and DC systems. The DC bus with a voltage level of $48 \mathrm{~V}$ is used to supply the Smart PoE Switches and the peripherals drivers that control the final actuators. We can distinguish 4 types of drivers:

- $\quad$ LED driver.

- Blind driver.

- HVAC driver (Valve and Modulating Flow Regulator (MFR))

- Multi-sensors driver.

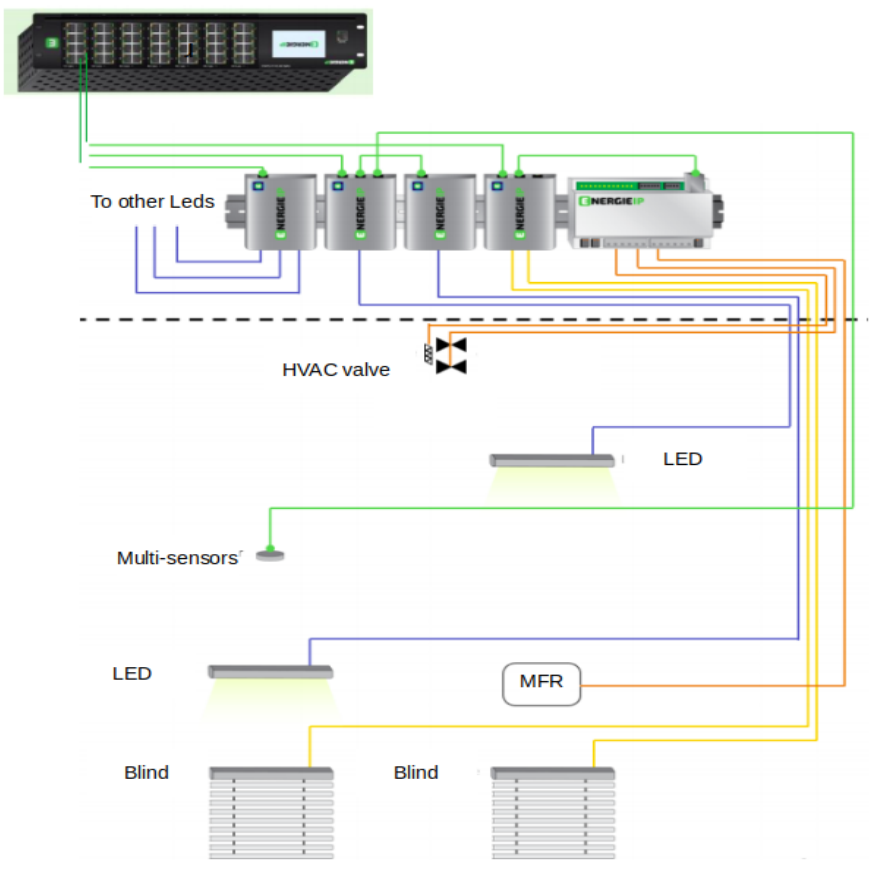

Fig. 3: Connection between a smart PoE Switch and the drivers [2]

As shown in Fig. 3, each Smart PoE switch has 56 ports with a total power of $5544 \mathrm{~W}$ and $99 \mathrm{~W}$ per single port. We have designed the PoE switch in a way that we can be able not only to manage the Ethernet packets but also to manage the quantity of energy supplied to each device by monitoring the PSEs. By this feature, we are able to manage in a better way the power bank of each switch and to route the energy to devices given certain features :

- Maximum Power.

- Minimum Power.

- Nominal Power.

- Priority.

- Niceness.

With the maximum and the minimum, we fix the power limits of each device, with the minimum power the device will be able to do the crucial minimum functions as communication and keep alive. The Nominal Power ensures a normal activity of the device by achieving all of its functions. Priority PR and niceness NI are two features used by the process scheduling in Linux. The scheduler is the core of the multitasking in Linux systems, using a priority-based scheduling algorithm to choose between the running processes in the system. It classifies processes based on the most deserving and the need for CPU time. Processes with a higher priority will be executed before those with a lower priority, while processes with the same priority are scheduled one after the next, repeatedly. In the Linux system, there are a total of 140 priorities and two distinct category ranges implemented. The first one is a nice value (niceness) which ranges from -20 (highest priority) to 19 (lowest priority) with 0 as the default value, the other is the realtime priority, which ranges from 1 to 99 by default, and from 100 to 139 for the user-space [9]. From a point of view of accessing to limited resources, the energy problem in building or in any power supply system with limited resources in general is similar to the problem of multi-tasks programs and accessing to the processor or memory which are limited resources in the computer science field, and that is why we propose these two features as main properties for our devices.

In order to reduce the energy consumption in buildings, we have to consider it as limited in resource even though buildings are usually connected to the Grid. Many studies have been done about energy management algorithms for buildings with renewable energy, all these studies have a major objective which is reducing the energy consumption from the Grid and favor the produced energy from renewable energy sources. A.Rifat Boynuegri et al. [10], in their study about energy management 
algorithm for smart home with renewable energy sources, proposed an algorithm based on the state of charge of the battery and the energy price from the Grid. The output of the algorithm is a priority level that reflects how we should manage the powered devices in each situation. The simulation had shown that with this algorithm, they could make about $28 \%$ of reduction in the electricity bill.

In this paper we don't study algorithms, we focus more on the importance of manageable connected devices and the need of routing the energy based on more intelligent factors and features like niceness and priority to better use this limited resource.

\section{Results and discussion}

In this project, we covered all the building surface by PoE devices, 72 smart PoE switches and more than 7000 drivers have been deployed. The switches had been placed in the building so as to respect a maximum distance allowed by the standard $802.3 \mathrm{bt}$ which is a distance of less than $100 \mathrm{~m}$.

After the deployment, and after doing the energy balance of the system, we noticed that the lines loss on the RJ45 cables are significant for the drivers placed in a distance greater than $30 \mathrm{~m}$ from the PoE switch. We have done more other intensive tests with a cable of $100 \mathrm{~m}$ that relay a LED driver with the switch. The results showed a loss of $20 \%$ for $40 \mathrm{~W}$ of transmitted power. The distance between the Smart PoE Switch and the drivers is a crucial parameter for the line energy losses, as shown in Fig. 4, we mainly consider 2 zones, zone one with an acceptable line energy losses which is less than $6 \%$ and zone two with an unacceptable line energy losses which is greater than $6 \%$ and can reach $20 \%$.

These results reveal that this architecture is suitable only for a small distance between the Smart PoE Switch and the drivers, a solution for this is to change the architecture by using smaller switches and get them closer to the drivers. For that and as a perspective of this part we propose another architecture based on 2 voltage levels 48 VDC and 380 VDC which has showed an effective results in terms of energy saving and reliability as presented in [4], [5], [6], [7] and [8]. In this section, we have presented our hybrid power supply system based on the PoE technology with the IEEE 802.3bt standard and we have discussed the benefits and the limits of this technology. We have also presented our energy routing system based on the communication between the Smart PoE Switches and the different drivers. In the next section, we are going to present our communication architecture, the chosen protocol, and its performances in a large scale deployment.

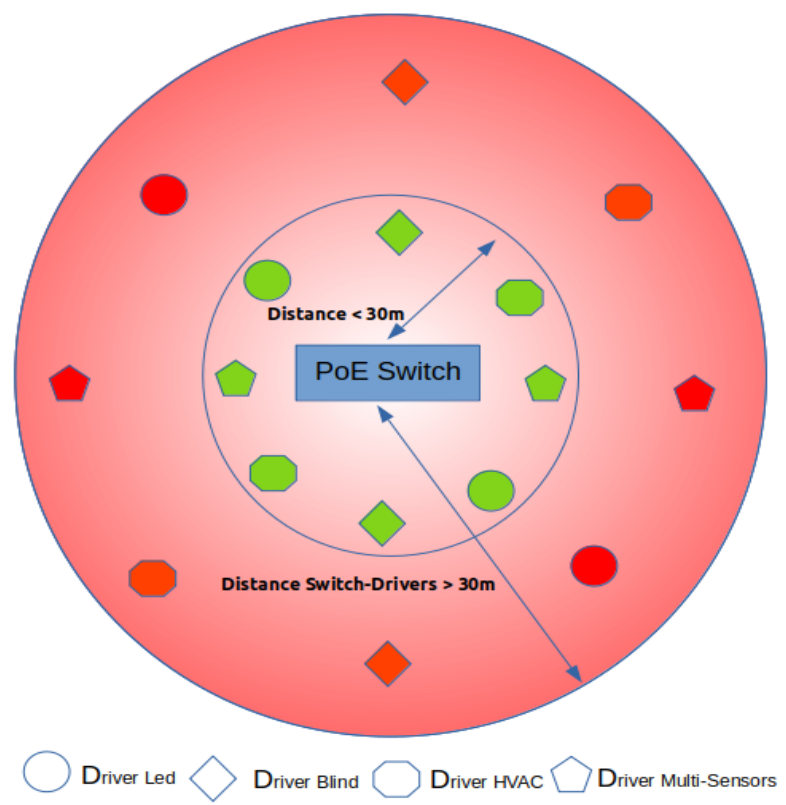

Fig. 4: Smart PoE switch and drivers distribution

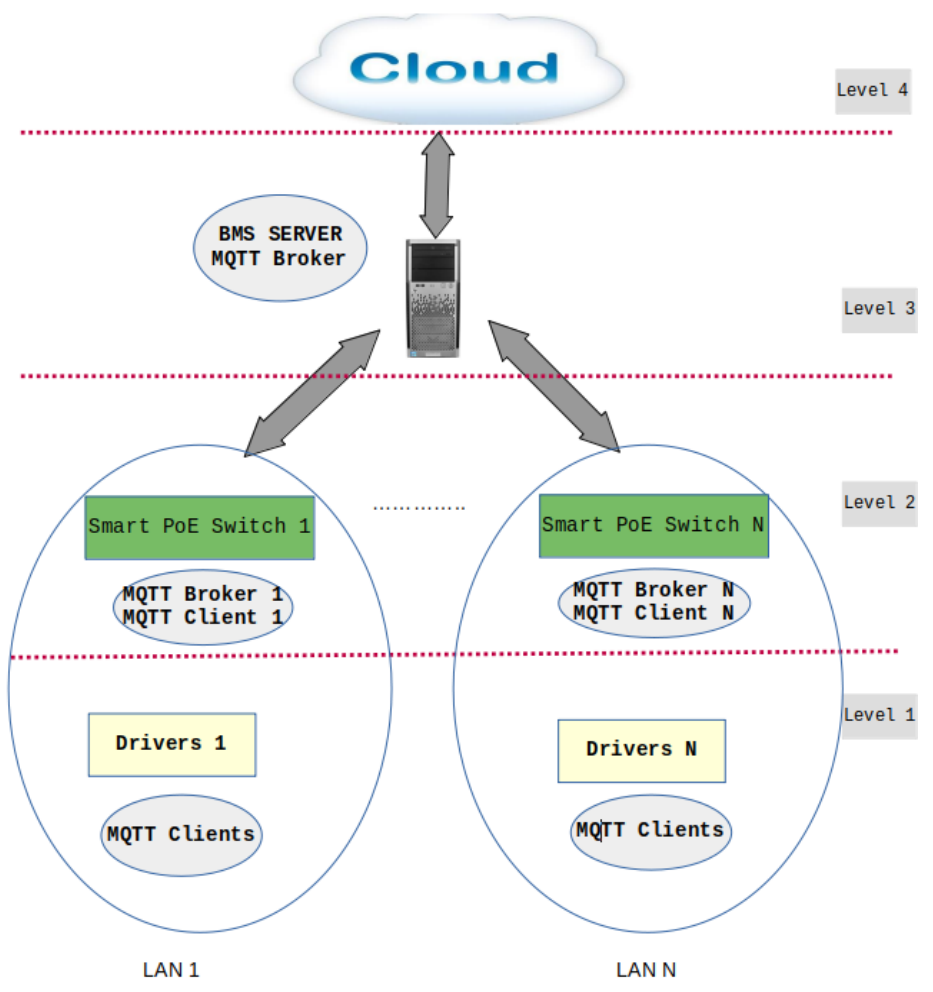

Fig. 5: Communication Architecture 


\section{Communication Architecture}

As shown in the Fig. 5, our network architecture is based on 4 levels of communication, each Smart PoE switch forms a separate local network with the different drivers connected to it. In this project, and with the $72 \mathrm{PoE}$ switches that cover all the building surface of $\left(23000 \mathrm{~m}^{2}\right)$, we formed 72 local networks. All the switches are assembled by 5 units in the form of patch bays, and form with the BMS server another local Network.

\section{A. Protocol choice}

The most studied and emerging messaging protocols for IoT systems are MQTT (Message Queuing Telemetry Transport), CoAP (Constrained Application Protocol), AMQP (Advanced Message Queuing Protocol), HTTP (Hypertext Transfer Protocol) and DPWS (Device Profile for Web Service). Many comparative studies of IoT protocols have been done such as [11], [12] and [13], and many criteria have been used to analyse the performances of each protocol like the standard, architecture, header sizes, quality of Service (QoS), transport protocol, security... These application protocols are different and each one has its advantages and its disadvantages, the choice of the application protocol depends mainly on the application itself and the constraints of the system. For a building management system, the main required criteria are classified as following :

- Reliability.

- Security.

- Scalability.

- Cost of hardware.

- Interoperability.

The Reliability in our system is the most crucial criterion especially on the level 2 of our architecture (Fig. 5), the level 1 presents the various local networks between each switch and its drivers. A non reliable communication at this level between a given smart PoE switch and its drivers cannot really cause a big problem because it can affect only some few devices, however a non reliable communication at the second level between the smart PoE switches and the BMS server can get down a whole branch of the system (at least 100 devices in our case). Based on the criteria cited before and the constraints of an industrial project, we have chosen MQTT as an application protocol for this project, and for the rest of this study we are going to analyse the performances of this protocol based on a real implementation.

\section{B. $M Q T T$}

MQTT is a Machine-to-Machine (M2M) data transfer protocol that enables systems to access IoT data. It is considered as one of the leading industrial Internet of Things (IIoT) messaging protocols. Indeed, even if there are multiple technologies and IIoT protocols, MQTT has succeeded due to its advantages:

- Reliable Message Delivery: MQTT is based on TCP as transport protocol which made it more reliable than all the application protocol based on UDP. Further more it uses 3 levels of quality of service (QoS) that boosts its reliability of message delivery.

- Secure: MQTT uses TLS to encrypt messages and can authenticate clients by using advanced authentication protocols such as OAuth.

- Scalable: MQTT can scale to connect millions of IoT devices.

- $\quad$ Lightweight and Efficient: MQTT clients are very small and require minimal resources, this proocol can so be used on microcontrollers with limited resources. MQTT message headers are also small which optimizes the network bandwidth.

MQTT have also some disadvantages which are mainly:

- Operates over TCP, the operation over TCP has the advantage of the reliability cited before but it has also a disadvantage for devices working on battery because TCP requires more handshaking to set up a communication and more in case of using TLS for security. The solution is to keep the session open which is also a huge problem for battery based application in term of energy consumption. In our case, this does not present a problem given that all our devices are working on PoE.

- Centralized broker: This can be a point of failure. A problem with the broker can affect the whole system.

\section{MQTT Delay}

A lot of studies have analysed MQTT performances. In this project we consider only the delay of an end-toend transmission (from the publisher to the subscriber) 
which is crucial in the case of our architecture and in the vision of the energy routing system that we propose: this routing system relies mainly on the network reactivity and the reliability of the network. Shinho Lee et al. [14] studied the end-to-end delay in wireless and wired MQTT network and its dependence on the QoS level and its correlation with packets loss. In the study of Deepsubhra Guha Roy et al. [15] about aplicationaware end-to-end delay and message loss estimation in Internet of Things, they had used the eclipse-paho to implement the MQTT-SN. These studies demonstrated the correlation between the end-to-end delay and the losses of packets and from the presented results, we can see that the delay in case of QoS2 is very high, higher than $100 \mathrm{~ms}$ for a 1000-byte message [14] and even higher than $300 \mathrm{~ms}$ in the study of [15]. These studies are done with a no secure MQTT without the TLS layer. In this study we are going to explain our method to evaluate MQTT end-to-end delay in the case of no secure and in the case of secure MQTT. We started our experimentation by evaluating a single node on the network, in order to measure the end-to-end delay the node publishes and describes to the same topic, it sends a first message and because it did already subscribed to that topic the broker will send it back, once received the node will start the same procedure for 100 times. We start a timer before sending the first message and we get the total time of 100 transmissions at the end of the procedure then we calculate the mean end-to-end delay by dividing the result by 100. Fig. 6 shows the results for secure MQTT and not secure MQTT in the case of QoS0 configuration. Fig. 7 shows the results in the case of QoS1 configuration. Either in the case of QoS0 or in the case of QoS1 we can see the influence of the TLS protocol and the delay which is minimal in the case of messages with minimal number of bytes but it get higher with the number of transmitted bytes, that can be explained by the equation that describes a packet delay along its route from source to destination:

$$
\begin{gathered}
\text { Delay }=\mathrm{D}_{\text {processing }}+D_{\text {queuing }}+D_{\text {transmission }}+ \\
D_{\text {propagation }}
\end{gathered}
$$

TLS protocol affects mainly the processing delay with the encryption and decryption times. We can also see the impact of the QoS level on the end-to-end delay by comparing Fig. 6 and 7. [13], [14] and [15] explained this impact by the correlation between the delay and the losses of packets but they didn't treat the affect of the number of nodes on the network which mainly affects the queuing delay and the technical features of the broker (processor, frequency...). P. Ferrari et al. [16] have studied the end-to-end delay with a hardwareindependent experiment and different MQTT brokers, the study has shown also the impact of the Round-Trip Delay (RTD) on the end-to-end delay. The RTD is neglected in our case since we are working in the case of a Local Network. We have done our tests on a real network with the presented devices in Table 2.

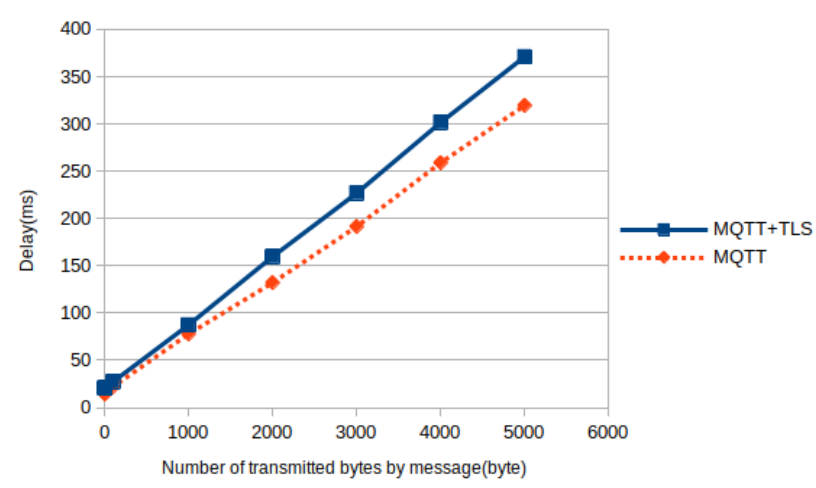

Fig. 6: MQTT End to End delay with QoS0

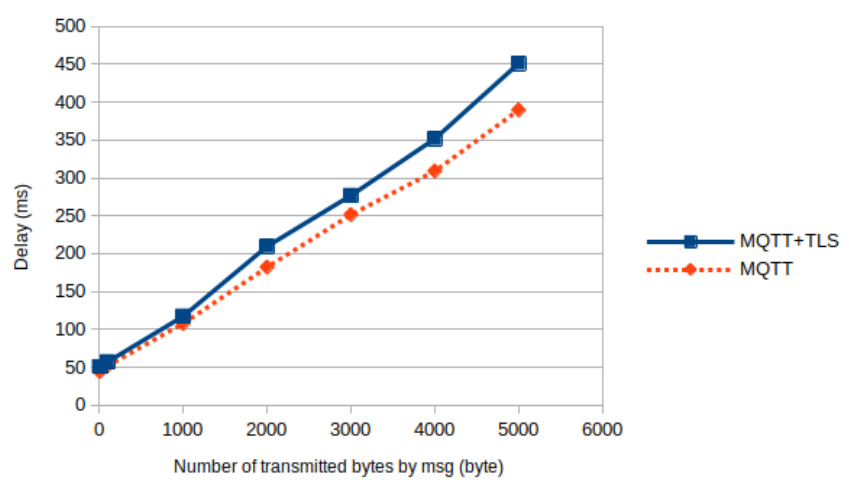

Fig. 7: MQTT End to End delay with QoS1

\begin{tabular}{|l|l|l|l|}
\hline & $\begin{array}{l}\text { Smart PoE } \\
\text { Switch }\end{array}$ & Drivers & BMS Server \\
\hline Processor & $\begin{array}{l}\text { BCM2837B0 } \\
\text { Cortex-A53 }\end{array}$ & Cortex-M4 & $\begin{array}{l}\text { BCM2837B0 } \\
\text { Cortex-A53 }\end{array}$ \\
\hline Frequency & $1.2 \mathrm{GHz}$ & $64 \mathrm{MHz}$ & $1.2 \mathrm{GHz}$ \\
\hline RAM & $1 \mathrm{Go}$ & $256 \mathrm{~KB}$ & $8 \mathrm{Go}$ \\
\hline ROM & $16 \mathrm{Go}$ & $1 \mathrm{MB}$ & $32 \mathrm{Go}$ \\
\hline
\end{tabular}

TABLE II: Features of the network nodes

The Network is composed of 74 devices as MQTT clients and one Broker, the results presented in Fig. 8 
show the delay difference between a network with only one client and a network with 74 clients. These results could be also affected by the activity of the other nodes on the network, in this experimentation all the other nodes were configured with a 1 second status report which means each device has to send its data at least once per second.

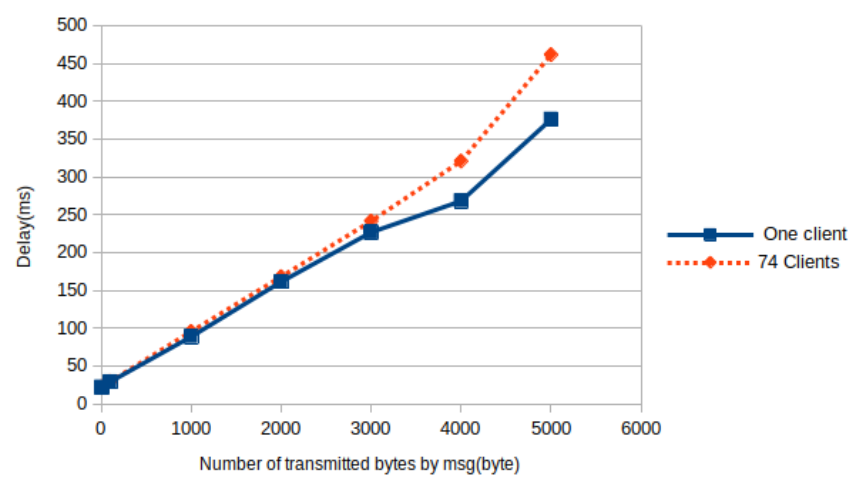

Fig. 8: Influence of the number of nodes on the end-toend Delay

\section{CONCLUSion}

In this study we have presented in a first part a DC power supply architecture based on the standard IEEE 802.3bt. We have discussed the benefits for enhanced energy management systems using smart PoE switches with ability to route energy to charges/drivers features under a global system energy constraints. We also have observed the limits of this architecture and the energy losses especially when using long cables between the PoE switches and the drivers. After the hardware, we have presented the communication architecture based on MQTT as an application protocol for interoperability, we have presented some of its features and we have analysed the delay of this protocol. MQTT highlighted a great reliability and stability in the Local network between the PoE switches and the drivers even the delay was acceptable given the few amounts of data exchange between devices on this level. The communication between the BMS server and the PoE switches uses much more data. On this level we consider that MQTT is not suitable for this task providing higher delay when arrived large data sets. In order to push toward positive energy buildings a perspective of this project, we will rectify the disadvantages of this system and we are developing a new architecture with 2 voltage level of 48 VDC (floor distribution) and 380 VDC (energy backbone) with a decentralised control/command system based on a distributed protocol on the second level, while keeping MQTT only for the first level of the actual architecture.

\section{ACKNOWLEDGEMENT}

This research is supported by an industrial grant of the French Gov ANRT supporting the research and developments for the French small company UBIANT. The authors want to thanks the staff and members of the Ubiant \& Energie IP companies for illustrations provided for this paper.

\section{REFERENCES}

[1] International Energy Agency, Online(2020) [Online]. Available: https://www.iea.org/data-andstatistics? country $=$ WORLDfuel $=\mathrm{CO} 2$

[2] International Energy Agency, Online(2020) [Online]. Available: https://www.iea.org/data-andstatistics?country $=$ WORLDfuel=Energy

[3] IEEE Draft Standard for Ethernet - Amendment 2: "Power over Ethernet over 4 Pairs", in IEEE P802.3bt/D3.6, June 2018, vol., no., pp.1-36, 1 Jan. 2018

[4] B. Raj Shrestha, Timothy M. Hansen; R. Tonkoski,"Reliability analysis of 380V DC distribution in data centers",IEEE Power \& Energy Society Innovative Smart Grid Technologies Conference (ISGT),Minneapolis, MN, USA, 6-9 Sept. 2016.

[5] H.Yajima, T. Babasaki, K. Usui ; Y. Ooi, K. Hirose, "Energysaving and efficient use of renewable energy by introducing an 380 VDC power-supply system in data centers", IEEE International Telecommunications Energy Conference (INTELEC), 18-22 Oct. 2015

[6] G. AlLee, W. Tschudi, "Edison Redux: 380 Vdc Brings Reliability and Efficiency to Sustainable Data Centers", IEEE Power and Energy Magazine, Volume: 10, Issue: 6, Nov.-Dec. 2012.

[7] D. J Becker, et B.J. Sonnenberg, "DC microgrids in buildings and data centers", IEEE International Telecommunications Energy Conference (INTELEC), Amsterdam, 9-13 Oct. 2011.

[8] Y. Yoshida, T. Hayashi, H. Kusabe, T. Kyunai, K. Usui, H. Yajima, K. Hirose, T. Aoki, D. Stanzione, 'Energy-saving effects by using $380 \mathrm{vdc}$ power supply system interconnected with a solar power generation system in texas",IEEE International Telecommunications Energy Conference (INTELEC), Broadbeach, QLD, Australia, 22-26 Oct. 2017.

[9] K. C. Wang, Chapter Process Management in Unix/Linux ,Systems Programming in Unix/Linux pp 101-14, 28 August 2018

[10] A. Rifat Boynuegri, B.Yagcitekin, M. Baysal, A. Karakas, M. Uzunoglu, "Energy management algorithm for smart home with renewable energy sources", in the 4th International Conference on Power Engineering, Energy and Electrical Drives, Istanbul, Turkey, 13-17 May 2013.

[11] S. Elhadi, A. Marzak, N. SAEL, S. MERZOUK, "Comparative study of IoT protocols", in the second International Conference on Smart Application and Data Analysis for Smart Cities, 12 Jun 2018. 
[12] N. Naik, "Choice of Effective Messaging Protocols for IoT Systems: MQTT, CoAP, AMQP and HTTP",in IEEE International Systems Engineering Symposium (ISSE), Vienna, Austria, October 2017.

[13] D. Thangavel, X. Ma, A. Valera, H. Tan, C. Keng-Yan TAN, "Performance Evaluation of MQTT and CoAP via a Common Middleware", in IEEE Ninth International Conference on Intelligent Sensors, Sensor Networks and Information Processing (ISSNIP) Symposium on Sensor Networks, Singapore, 21-24 April 2014.

[14] S. Lee, H. Kim, D. Hong, H. Ju, "Correlation Analysis of MQTT Loss and Delay According to QoS Level". in the International Conference on Information Networking 2013 (ICOIN), Bangkok, Thailand, 28-30 January, 2013.

[15] D. Guha Roy, B. Mahato , D. De , R. Buyya , "Applicationaware end-to-end delay and message loss estimation in Internet of Things (IoT) - MQTT-SN protocols",in Future Generation Computer Systems Volume 89, pages 300-316, December 2018.

[16] P. Ferrari, A. Flammini, E. Sisinni, S. Rinaldi, D. Brandão and M. S. Rocha, "Delay Estimation of Industrial IoT Applications Based on Messaging Protocols," in IEEE Transactions on Instrumentation and Measurement, vol. 67, no. 9, pp. 2188-2199, Sept. 2018. 